\title{
Clinical and Pathological Correlation in Pediatric Invasive Pulmonary Aspergillosis
}

\author{
Nattachai Anantasit ${ }^{1,2 *}$, Noramon Nuntacharruksa1, Pimpin Incharoen ${ }^{3}$ \\ and Aroonwan Preutthipan ${ }^{1}$
}

'Division of Pediatric Pulmonology, Department of Pediatric, Faculty of Medicine, Ramathibodi Hospital, Mahidol University, Bangkok, Thailand, '2Division of Pediatric Critical Care, Department of Pediatric, Faculty of Medicine, Ramathibodi Hospital, Mahidol University, Bangkok, Thailand, ${ }^{3}$ Department of Pathology, Faculty of Medicine, Ramathibodi Hospital, Mahidol University, Bangkok, Thailand

Introduction: Invasive' pulmonary aspergillosis (IPA) has been one of the major causes of mortality in immunocompromised patients. The gold standard method for a diagnosis of IPA is histopathological examination of the lung tissue; however, post-procedural bleeding limits the feasibility of lung biopsy. The European Organization for Research and Treatment of Cancer/Invasive Fungal Infections Cooperative Group and The National Institute of Allergy and Infectious Disease Mycoses Study Group (EORTC/MSG) defined IPA. The objective of this study was to validate the EORTC/MSG 2008 definition of IPA, compared with histopathology in the pediatric population.

OPEN ACCESS

Edited by: Yves Ouellette, Mayo Clinic, United States

Reviewed by: Yee Hui Mok,

KK Women's and Children's Hospital, Singapore Sameer Shantaram Kamath, Duke Children's Hospital \& Health

Center, United States

${ }^{*}$ Correspondence: Nattachai Anantasit nattachai032@hotmail.com

Specialty section: This article was submitted to Pediatric Critical Care, a section of the journal Frontiers in Pediatrics

Received: 04 November 2017 Accepted: 05 February 2018 Published: 21 February 2018

Citation: Anantasit N, Nuntacharruksa N, Incharoen $P$ and Preutthipan A (2018) Clinical and Pathological Correlation in Pediatric Invasive Pulmonary Aspergillosis. Front. Pediatr. 6:31. doi: 10.3389/fped.2018.00031
Methods: Histopathological examinations of lung tissues of children aged 1 month18 years with respiratory tract infection at the time of obtaining biopsy were retrieved. Retrospective chart reviews for clinical characteristics were performed. IPA diagnosis was classified according to the EORTC/MSG 2008 definition.

Results: During the 10-year period, there were 256 lung tissues, of which 58 specimens were suspected to have pulmonary infection. Fourteen patients (24\%) were noted to have IPA. Seven patients (50\%) with proven IPA were classified as probable, while the remaining 50\% were classified as possible, and none were classified as no IPA, by using EORTC/MSG 2008 definition. Other 44 specimens demonstrated 14 (32\%), 14 (32\%), and 16 (36\%) were classified as probable, possible, and no IPA, respectively. When comparing probable or possible IPA with no IPA, we found that the EORTC/MSG 2008 definition had $100 \%$ sensitivity, 36\% specificity, 33\% positive predictive value, and 100\% negative predictive value in diagnosis of IPA.

Conclusion: Our study illustrated that the EORTC/MSG 2008 definition provided an excellent sensitivity but low specificity for diagnosing IPA.

Keywords: invasive pulmonary aspergillosis, pediatric, histopathology, definition, sensitivity

\section{INTRODUCTION}

Invasive pulmonary aspergillosis (IPA) has been one of the major causes of mortality in immunocompromised patients, such as malignancy, hematopoietic stem cell transplantation, and prolonged usage of immunosuppressive agents. Early diagnosis and prompt treatment improve survival outcome $(1,2)$. Aspergillus species are ubiquitous in the environment. Aspergillus fumigatus is the most common species in IPA $(3,4)$. Other species include Aspergillus flavus, Aspergillus niger, and Aspergillus 
terreus. Aspergillus is introduced to the lower respiratory tract by inhalation of the infectious spores. In healthy hosts, spores are eliminated by mucociliary clearance and immune defense. In immunocompromised patients, dormant spores convert into growing hyphal elements and invade lung parenchyma and vascular structure (1). Clinical symptoms and signs of IPA are indistinguishable from other pathogens causing pneumonia. Chest radiography is also non-specific. Therefore, the diagnostic tool is challenging $(5,6)$.

The gold standard method in the diagnosis of IPA is histopathological examination of lung tissue obtained by bronchoscopy or open thoracotomy. The presence of angioinvasion by acute angle, branching septate fungal hyphae along with a positive culture for Aspergillus from the same site is diagnostic for IPA (1). However, invasive procedures hinder the possibility of obtaining lung tissues. The angioinvasive nature of Aspergillus and the patient condition such as thrombocytopenia further increase risk of bleeding and other complications.

In 2002, The European Organization for Research and Treatment of Cancer/Invasive Fungal Infections Cooperative Group and The National Institute of Allergy and Infectious Disease Mycoses Study Group (EORTC/MSG) formed a consensus committee to develop a standard definition for invasive fungal infections, including IPA. The definitions were intended for use in the context of clinical and/or epidemiological research, not for clinical decision making (7). These definitions were revised in 2008 due to the advances in diagnostic tools, including galactomannan, beta-D-glucan, and fungal DNA in body fluids by PCR $(8,9)$. Diagnosis of IPA is challenging, as the clinical symptoms are not specific. There is no single investigation for the diagnosis. IPA carries a high mortality rate if untreated, and delayed treatment can result in death. The ideal diagnostic tool should have high sensitivity for early recognition and treatment. In brief, based on EORTC/MSG 2008 consensus definition, there were three classifications; proven, probable, and possible. Proven IPA requires histopathology with a characteristic of infection and/or a positive culture of specimen from a sterile site. Probable IPA requires the fulfillment of criteria within three categories: host factors, clinical, and mycological criteria. Possible IPA consists of host factors, and clinical criteria without mycological evidence of Aspergillus infection (8). These definitions have been widely used in epidemiological research, and clinical trials for evaluation of new drugs and management strategies. However, they were based on the literature review, which did not include pathological results and was originally for the adult oncological population. To our knowledge, there were limited data about validity of the consensus definition compared with the histopathological diagnosis of IPA especially in the pediatric population. The objective of this study was to validate the EORTC/MSG 2008 consensus definition with the gold standard of histopathological results in pediatric patients.

\section{MATERIALS AND METHODS}

\section{Study Design and Purpose}

This study was performed in a tertiary care, academic center. We retrospectively analyzed histopathology tissues in children aged 1 month-18 years with clinical suspicion of respiratory tract infection from January 2006 to December 2016. This study was approved by the Institutional Review Board. Patients with congenital lung disease, pulmonary malignancy, and incomplete medical records were excluded. Clinical characteristics, diagnostic tests, treatments, and pathological results were reviewed. Patients were categorized into two groups by the histopathological result as proven IPA and non-proven IPA and were classified according to the EORTC/MSG 2008 consensus definition into three groups (probable, possible, and no IPA) as shown in Figure 1.

\section{Definitions}

The definition criteria for proven, probable, and possible IPA were shown in Table $\mathbf{1 .}$

Proven IPA is characterized by a discrete nodule consisting of necrotic lung tissue with angioinvasion by acute angle, branching septate fungal hyphae or culture positive for Aspergillus spp. from lung tissue specimen $(10,11)$.

Non-proven IPA is defined as patients who do not meet the pathological criteria.

According to the EORTC/MSG 2008 consensus definition (8), clinical criteria in IPA consists of the presence of one of the following three signs on computer tomography; dense, wellcircumscribed lesions with or without a halo sign, air-crescent sign, or cavity.

Positive clinical IPA is defined as patients who have clinical diagnosis with probable or possible IPA by the EORTC/MSG 2008 consensus definition.

Negative clinical IPA is defined as patients who do not meet criteria for diagnosis by the EORTC/MSG 2008 consensus definition.

Galactomannan enzyme immunoassay was measured from serum and bronchoalveolar lavage (BAL) fluid. Results were recorded as an index relative to the mean optical density of the threshold controls (GM index = optical density sample/mean optical density of the threshold control samples). Samples that had an index value $>0.5$ were considered positive for both serum and BAL fluid specimen $(12,13)$.

\section{Statistical Methods}

SPSS software (version 18.0, SPSS Inc., Chicago, IL, USA) was used for statistical analysis. Patients' demographics, clinical characteristics, and pathological data were analyzed by descriptive analysis using student's t-test and Chi-Square. For the diagnostic test, sensitivity, specificity, positive predictive value, and negative predictive value were obtained. Correlation between clinical diagnosis and pathological result was calculated using Spearman rho correlation analysis. A p-value of less than 0.05 was considered statistically significant.

\section{RESULTS}

In total, 256 lung tissues were documented. Fifty-eight pathological specimens revealed suspicion of infectious etiologies, $14(24 \%)$ patients were consistent with proven IPA, and 44 (76\%) patients were non-proven IPA as shown in Figure 1. All 


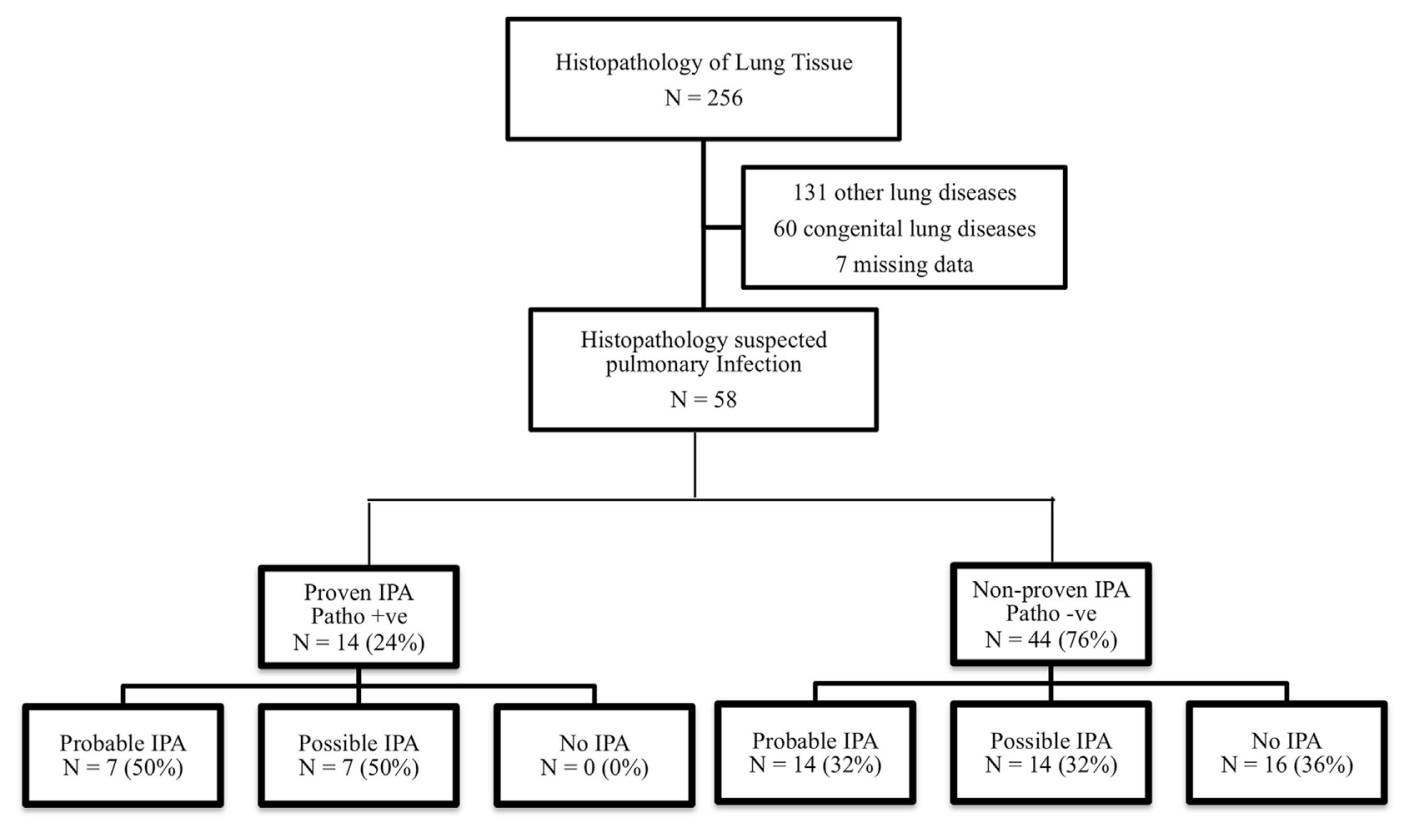

FIGURE 1 | Flow diagram and classification of invasive pulmonary aspergillosis (IPA) according to the EORTC/MSG 2008 consensus definition.

TABLE 1 | The diagnostic criteria for IPA according to the EORTC/MSG 2008 consensus definition (8).

\begin{tabular}{|c|c|c|c|}
\hline & $\begin{array}{c}\text { Proven } \\
\text { IPA }\end{array}$ & $\begin{array}{c}\text { Probable } \\
\text { IPA }\end{array}$ & $\begin{array}{c}\text { Possible } \\
\text { IPA }\end{array}$ \\
\hline $\begin{array}{l}\text { Positive for histopathological criteria } \\
\text { - A discrete nodule consisting of } \\
\text { necrotic lung tissue with angioinvasive } \\
\text { by acute angle branching septate fungal } \\
\text { hyphae or culture positive for Aspergillus } \\
\text { spp. from lung tissue specimen }\end{array}$ & Yes & No & No \\
\hline $\begin{array}{l}\text { Positive for host factor criteria } \\
\text { - Neutropenia } \\
\text { - Post stem cell transplantation } \\
\text { - Prolonged corticosteroid use } \\
\text { - Received immunosuppressants } \\
\text { - Inherited immunodeficiency }\end{array}$ & & Yes & Yes \\
\hline $\begin{array}{l}\text { Positive for clinical criteria } \\
\text { Presence of } 1 \text { of the following } \\
\text { three signs on CT } \\
\text { - Dense, well-circumscribed lesion } \\
\text { with or without a halo sign } \\
\text { - Air-crescent sign } \\
\text { - Cavity }\end{array}$ & & Yes & Yes \\
\hline $\begin{array}{l}\text { Positive for mycological criteria } \\
\text { - Positive direct test by culture } \\
\text { - Positive indirect tests by } \\
\quad \text { galactomannan antigen detection }\end{array}$ & & Yes & No \\
\hline
\end{tabular}

IPA; invasive pulmonary aspergillosis, CT; computer tomography.

patients who were diagnosed with probable or possible IPA by the EORTC/MSG 2008 definition (positive clinical IPA) received antifungal medications, although 28 out of 42 patients were not diagnosed with proven IPA, but received antifungal medications. Five of 16 patients who were not diagnosed with IPA by the
EORTC/MSG 2008 definition (negative clinical IPA) received antifungal medications. None of these patients were diagnosed with proven IPA. The adverse events from antifungal medications were hypokalemia and acute kidney injury, although none of patients required renal replacement therapy. Baseline characteristics of histopathological proven IPA and non-proven IPA were showed in Table 2. There was no significant difference of demographic data in both groups. The proven IPA group had significantly more neutropenic patients than the non-proven IPA group ( $\mathrm{p}$-value $=0.009$ ). The mean duration of neutropenia was longer in proven IPA than in non-proven IPA group. Mean was $22.1 \pm 35.3$ days in proven IPA and $12.9 \pm 12.5$ days in nonproven IPA group $(\mathrm{p}$-value $=0.307)$.

\section{Microbiology and Pathological Results}

Of the 58 lung tissue specimens, 18 lung tissues were obtained by open biopsy, 13 by necropsy, 10 by autopsy, 10 by CT/US guided needle biopsy, and 7 by transbronchial biopsy. The reasons for biopsy were for diagnosis in $28(48 \%)$ patients, no response to antifungal medications or progression of diseases in 20 (35\%) patients, and others in $10(17 \%)$ patients. The mean duration from treatment to biopsy was 20.6 and 35.9 days in proven and non-proven IPA group, respectively ( $\mathrm{p}$-value $=0.431$ ). The microbiology in non-proven IPA was virus in 7 patients (cytomegalovirus in 6 cases and respiratory syncytial virus in 1 case) and bacteria were found in 10 patients ( 3 cases were A. baumannii, 2 cases P. aeruginosa, 2 cases Actinomycoses spp., 1 case M. tuberculosis, and 2 cases other bacteria). Fungus was isolated in nine patients (four of Mucormycosis spp., two of Cryptococcosis spp., two of Pneumocystis spp., and 1 of Scedosporiosis spp.). The remaining 12 patients showed histopathologic change without identified pathogens, such as diffused alveolar damage and 
TABLE 2 | Baseline characteristics of the included patients.

\begin{tabular}{|c|c|c|c|}
\hline & $\begin{array}{c}\text { Proven } \\
\text { IPA }(n=14)\end{array}$ & $\begin{array}{l}\text { Non-proven } \\
\text { IPA }(n=44)\end{array}$ & $p$-Value \\
\hline Age, mean $\pm S D$ & $10.4 \pm 5.9$ & $9.0 \pm 6.1$ & 0.438 \\
\hline Male (\%) & $4(29)$ & $21(48)$ & 0.207 \\
\hline Underlying disease, $n$ (\%) & & & 0.064 \\
\hline - Malignancy & $12(86)$ & $19(43)$ & \\
\hline - Primary immune deficiency & $0(0)$ & $6(14)$ & \\
\hline - Connective tissue disease & $0(0)$ & $3(7)$ & \\
\hline - Others & $2(14)$ & $9(20)$ & \\
\hline - Healthy & $0(0)$ & $7(16)$ & \\
\hline Neutropenia, $n(\%)$ & $11(79)$ & $17(39)$ & $0.023^{*}$ \\
\hline Post transplantation, $n$ (\%) & $1(7)$ & $6(14)$ & 0.108 \\
\hline Corticosteroid use, $n(\%)$ & $1(7)$ & $4(9)$ & 0.904 \\
\hline Presentation symptom, $n$ (\%) & & & 0.343 \\
\hline - Febrile neutropenia & $9(64)$ & $18(41)$ & \\
\hline - Dyspnea & $1(7)$ & $10(23)$ & \\
\hline - Hemoptysis & $2(14)$ & $7(16)$ & \\
\hline - Others & $2(14)$ & $9(20)$ & \\
\hline Serum galactomannan, median (IQR) & $0(0-2.2)$ & $0(0-1.4)$ & 0.889 \\
\hline BAL galactomannan, median (IQR) & $3.4(1.5-7.5)$ & $2.3(0-4.0)$ & 0.772 \\
\hline Computer tomography finding, $n(\%)$ & & & 0.390 \\
\hline $\begin{array}{l}\text { - Dense, well-circumscribed with/ } \\
\text { without Halo sign }\end{array}$ & $11(79)$ & $26(59)$ & \\
\hline - Non-specific infiltration/consolidation & $2(14)$ & $9(21)$ & \\
\hline - Air-crescent sign & $1(7)$ & $1(2)$ & \\
\hline - Cavitation & $0(0)$ & $7(16)$ & \\
\hline - Normal finding & $0(0)$ & $1(2)$ & \\
\hline Exposure to beta-lactam antibiotics, $n(\%)$ & $2(14.3)$ & $10(22.7)$ & 0.497 \\
\hline Antifungal treatment, $n(\%)$ & & & 0.200 \\
\hline - Amphotericin B & $6(43)$ & $22(50)$ & \\
\hline - Combination drugs & $4(29)$ & $4(9)$ & \\
\hline - Voriconazole & $3(21)$ & $6(14)$ & \\
\hline - Caspofungin & $1(7)$ & $1(2)$ & \\
\hline - No antifungal & $0(0)$ & $11(25)$ & \\
\hline Hospital mortality, $n(\%)$ & $6(43)$ & $23(52)$ & 0.539 \\
\hline
\end{tabular}

IPA; invasive pulmonary aspergillosis, IQR; interquartile range.

*p-Value less than 0.05 .

pulmonary hemorrhage, and 6 patients showed non-infectious histopathology.

\section{EORTC/MSG 2008 Consensus Definition}

We analyzed clinical diagnosis of IPA by grouping probable and possible IPA as positive clinical IPA, and no IPA as negative clinical IPA. The sensitivity was $100 \%$ and the specificity was $36 \%$. The positive and negative predictive values were 33 and $100 \%$, respectively. Spearman's rho correlation analysis revealed a correlation between clinical diagnosis and pathological result $(\mathrm{r}=0.35$, $\mathrm{p}$-value $=0.008)$. Table 3 demonstrated each element in the EORTC/MSG 2008 consensus definition, in terms of sensitivity, specificity, positive, and negative predictive values.

\section{DISCUSSION}

This study validated the EORTC/MSG 2008 consensus definition with the gold standard histopathological tissue in pediatric population. Our results were consistent with previous studies revealing that proven IPA patients were found to be more neutropenic patients. The function of neutrophil is directed against Aspergillus hyphae (14). The neutropenia can increase risk of
TABLE 3 | The sensitivity and specificity of each element in the EORTC/MSG 2008 consensus definition.

\begin{tabular}{lcccc}
\hline & $\begin{array}{c}\text { Sensitivity } \\
(\mathbf{\%})\end{array}$ & $\begin{array}{c}\text { Specificity } \\
\mathbf{( \% )}\end{array}$ & $\begin{array}{c}\text { PPV } \\
\mathbf{( \% )}\end{array}$ & $\begin{array}{c}\text { NPV } \\
\mathbf{( \% )}\end{array}$ \\
\hline - Host risk & 100 & 15.9 & 27.5 & 100 \\
- Computer & 85.7 & 22.7 & 26.1 & 83.3 \\
$\quad$ tomography finding & & & & \\
- Culture & 36.4 & 100 & 100 & 81.6 \\
- Serum galactomannan & 33.3 & 68.2 & 22.2 & 79 \\
- BAL galactomannan & 100 & 26.7 & 26.7 & 100 \\
\hline
\end{tabular}

PPV; positive predictive value, NPV; negative predictive value, BAL; bronchoalveolar lavage.

IPA. A prior study showed that cavitation or air-crescent sign in chest computed tomography is helpful in diagnosing IPA (15). Nevertheless, pediatric studies have demonstrated that nodules or infiltration are the most common radiologic findings in pediatric IPA $(16,17)$. Our study found no difference in radiologic findings in proven IPA and non-proven IPA groups. We had defined positive imaging as dense, well-circumscribed lesions with or without halo sign, air-crescent sign, or cavitation. The analysis showed high sensitivity but low specificity as in a previous study (18).

Galactomannan is a cell wall polysaccharide released by Aspergillus species during growth $(19,20)$. Serum galactomannan is more superior than BAL fluid because it is less invasive and provides a higher sensitivity (21). However, our study illustrated that the sensitivity of serum galactomannan was lower than BAL (33 vs. $100 \%$, respectively) which was a similar result to another study (22). We hypothesized that since lungs are a primary site of IPA before angioinvasion may causes an elevation of BAL galactomannan prior to that of serum (23). In our study, the specificity of BAL fluid galactomannan was surprisingly low (27\%) compared with previous studies (87.5-94\%) using the same cutoff value $(20,24,25)$. However, this study had included non-malignant patients which was different from the previous studies. In addition, this could be explained by using different classification as most studies used proven and probable as IPA instead of using histopathology as a gold standard. Another reason could be from the beta-lactam antibiotics. The beta-lactam antibiotics are derived from molds of the genus Penicillium. Galactomannan moieties are shared between Aspergillus and Penicillium species (26). Therefore, beta-lactam antibiotics have been reported to cause false positive of galactomannan.

To our knowledge, this study is the first study to validate the EORTC/MSG 2008 consensus definition in pediatric population with IPA by using histopathological tissues. Although the EORTC/MSG 2008 consensus definition was originally for the adult immunocompromised population. This study showed that the EORTC/MSG 2008 consensus definition had 100\% sensitivity, but low specificity (36\%), in diagnosing IPA when compared with histopathology in pediatric population. IPA has high mortality if left untreated, our paper showed the EORTC/MSG 2008 consensus definition can be helpful as the screening tool in pediatric populations. Therefore, children with probable or possible IPA according to this definition should be promptly treated with antifungal medications, monitored for adverse events of 
antifungal medications, and need confirmed diagnosis if there is no clinical response.

This study has some limitations. First, retrospective study might not provide all data needed especially serum and BAL galactomannan. Second, the prevalence of IPA might be underestimated because our study started with histopathological tissue, as we were unable to perform biopsy in every case of suspected IPA. In addition, some patients only underwent imaging guided biopsy or transbronchial biopsy, which might result in inadequate specimen. Therefore, it may not represent all pediatric IPA. The last limitation is that this was a single-center study with small sample size. A larger sample size of these groups would allow better results and analysis.

\section{CONCLUSION}

Pediatric IPA is a life-threatening disease with high mortality. The diagnostic tool is an important method to early recognition and prompt treatment. Our study shows that the EORTC/MSG 2008 consensus definition provides an excellent sensitivity as screening tool, but low specificity for the diagnosis of IPA.

\section{ETHICS STATEMENT}

This study was carried out in accordance with the recommendations of Committee on human rights related to research involving

\section{REFERENCES}

1. Patterson KC, Strek ME. Diagnosis and treatment of pulmonary aspergillosis syndromes. Chest (2014) 146:1358-68. doi:10.1378/chest.14-0917

2. Steinbach WJ. Invasive aspergillosis in pediatric patients. Curr Med Res Opin (2010) 26:1779-87. doi:10.1185/03007995.2010.487793

3. Perfect JR, Cox GM, Lee JY, Kauffman CA, de Repentigny L, Chapman SW, et al. The impact of culture isolation of Aspergillus species: a hospital-based survey of aspergillosis. Clin Infect Dis (2001) 33:1824-33. doi:10.1086/ 323900

4. Thammahong A, Thayidathara $\mathrm{P}$, Suksawat $\mathrm{K}$, Chindamporn A. Invasive Aspergillus infections in a Thai tertiary-care hospital during 2006-2011. Adv Microbiol (2015) 5:298-306. doi:10.4236/aim.2015.55029

5. Kousha M, Tadi R, Soubani AO. Pulmonary aspergillosis: a clinical review. Eur Respir Rev (2011) 20:156-74. doi:10.1183/09059180.00001011

6. Thomas KE, Owens CM, Veys PA, Novelli V, Costoli V. The radiological spectrum of invasive aspergillosis in children: a 10-year review. Pediatr Radiol (2003) 33:453-60. doi:10.1007/s00247-003-0919-4

7. Ascioglu S, Rex JH, de Pauw B, Bennett JE, Bille J, Crokaert F, et al. Defining opportunistic invasive fungal infections in immunocompromised patients with cancer and hematopoietic stem cell transplants: an international consensus. Clin Infect Dis (2002) 34:7-14. doi:10.1086/323335

8. De Pauw B, Walsh TJ, Donnelly JP, Stevens DA, Edwards JE, Calandra T, et al. Revised definitions of invasive fungal disease from the European Organization for Research and Treatment of Cancer/Invasive Fungal Infections Cooperative Group and the National Institute of Allergy and Infectious Disease Mycoses Study Group (EORTC/MSG) consensus group. Clin Infect Dis (2008) 46:1813-21. doi: $10.1086 / 588660$

9. Tsitsikas DA, Morin A, Araf S, Murtagh B, Johnson G, Vinnicombe S, et al. Impact of the revised (2008) EORTC/MSG definitions for invasive fungal disease on the rates of diagnosis of invasive aspergillosis. Med Mycol (2012) 50:538-42. doi:10.3109/13693786.2011.630040

10. Tochigi N, Okubo Y, Ando T, Wakayama M, Shinozaki M, Gocho K, et al. Histopathological implications of Aspergillus infection in lung. Mediators Inflamm (2013) 2013:809798. doi:10.1155/2013/809798 human subjects, Faculty of Medicine Ramathibodi Hospital, Mahidol University.

\section{AUTHOR CONTRIBUTIONS}

NN and NA contributed to design of the study, data collection, data analysis, and manuscript drafting. PI and AP contributed to design of the study. NA critically revised it for important intellectual content. All authors gave final approval of the version to be published.

\section{ACKNOWLEDGMENTS}

The authors would like to acknowledge the departments of pediatric and pathology of Ramathibodi Hospital, Mahidol University that allowed us to analyze this project. We would like to thank Dr. Mahippathorn Chinnapha, Associate Professor Pornthep Tanpowpong, and Mr. Stephen Pinder for grammar correction. Furthermore, our special thanks to all patients who were recruited into this study.

\section{FUNDING}

This work did not receive any specific grant from funding agencies in the public, commercial, or not-for-profit sectors.

11. Hope WW, Walsh TJ, Denning DW. The invasive and saprophytic syndromes due to Aspergillus spp. Med Mycol (2005) 43:207-38.

12. Marr KA, Balajee SA, McLaughlin L, Tabouret M, Bentsen C, Walsh TJ. Detection of galactomannan antigenemia by enzyme immunoassay for the diagnosis of invasive aspergillosis: variables that affect performance. J Infect Dis (2004) 190:641-9. doi:10.1086/422009

13. Pfeiffer CD, Fine JP, Safdar N. Diagnosis of invasive aspergillosis using a galactomannan assay: a meta-analysis. Clin Infect Dis (2006) 42:1417-27. doi:10.1086/503427

14. Berenguer J, Allende MC, Lee JW, Garrett K, Lyman C, Ali NM, et al. Pathogenesis of pulmonary aspergillosis: granulocytopenia versus cyclosporine and methylprednisolone-induced immune suppression. Am J Respir Crit Care Med (1995) 152:1079-86. doi:10.1164/ajrccm.152.3.7663787

15. Gefter WB, Albelda SM, Talbot GH, Gerson SL, Cassileth PA, Miller WT Invasive pulmonary aspergillosis and acute leukemia. Limitations in the diagnostic utility of the air crescent sign. Radiology (1985) 157:605-10. doi:10.1148/radiology.157.3.4059547

16. Burgos A, Zaoutis TE, Dvorak CC, Hoffman JA, Knapp KM, Nania JJ, et al. Pediatric invasive aspergillosis: a multicenter retrospective analysis of 139 contemporary cases. Pediatrics (2008) 121:e1286-94. doi:10.1542/ peds.2007-2117

17. Anugulruengkitt S, Trinavarat $\mathrm{P}$, Chantranuwat $\mathrm{P}$, Sritippayawan $\mathrm{S}$, Pancharoen C, Thanyawee P. Clinical features and survival outcomes of invasive aspergillosis in pediatric patients at a medical school in Thailand. J Med Assoc Thai (2016) 99:150-8.

18. Blot SI, Taccone FS, Van den Abeele AM, Bulpa P, Meersseman W, Brusselaers N, et al. A clinical algorithm to diagnose invasive pulmonary aspergillosis in critically ill patients. Am J Respir Crit Care Med (2012) 186:56-64. doi:10.1164/rccm.201111-1978OC

19. Klont RR, Mennink-Kersten MA, Verweij PE. Utility of Aspergillus antigen detection in specimens other than serum specimens. Clin Infect Dis (2004) 39:1467-74. doi:10.1086/425317

20. Guo YL, Chen YQ, Wang K, Qin SM, Wu C, Kong JL. Accuracy of BAL galactomannan in diagnosing invasive aspergillosis: a bivariate metaanalysis and systematic review. Chest (2010) 138:817-24. doi:10.1378/chest.10-0488 
21. Salonen J, Lehtonen OP, Terasjarvi MR, Nikoskelainen J. Aspergillus antigen in serum, urine, and bronchoalveolar lavage specimens of neutropenic patients in relation to clinical outcome. Scand J Infect Dis (2000) 32:485-90. doi:10.1080/003655400458749

22. Zou M, Tang L, Zhao S, Zhao Z, Chen L, Chen P, et al. Systematic review and meta-analysis of detecting galactomannan in bronchoalveolar lavage fluid for diagnosing invasive aspergillosis. PLoS One (2012) 7:e43347. doi:10.1371/ journal.pone.0043347

23. Heng SC, Morrissey O, Chen S. Utility of bronchoalveolar lavage fluid galactomannan alone or in combination with PCR for the diagnosis of invasive aspergillosis in adult hematology patients: a systemic review and meta-analysis. Crit Rev Microbiol (2015) 41:124-34. doi:10.3109/1040841X.2013.804033

24. de Mol M, de Jongste JC, van Westreenen M, Merkus PJ, de Vries AH, Hop WC, et al. Diagnosis of invasive pulmonary aspergillosis in children with bronchoalveolar lavage galactomannan. Pediatr Pulmonol (2013) 48:789-96. doi:10.1002/ppul.22670

25. Mohammadi S, Khalilzadeh S, Goudarzipour K, Hassanzad M, Mahdaviani A, Aarabi N, et al. Bronchoalveolar galactomannan in invasive pulmonary aspergillosis: a prospective study in pediatric patients. Med Mycol (2015) 53:709-16. doi:10.1093/mmy/myv053

26. Ansorg R, van den Boom R, Rath PM. Detection of Aspergillus galactomannan antigen in foods and antibiotics. Mycoses (1997) 40:353-7. doi:10.1111/j. 1439-0507.1997.tb00249.x

Conflict of Interest Statement: All authors: there are no potential conflicts of interest. All authors have submitted the ICMJE Form for Disclosure of Potential Conflicts of Interest. Conflicts that the editors consider relevant to the content of the manuscript have been disclosed.

Copyright (c) 2018 Anantasit, Nuntacharruksa, Incharoen and Preutthipan. This is an open-access article distributed under the terms of the Creative Commons Attribution License (CC BY). The use, distribution or reproduction in other forums is permitted, provided the original author(s) and the copyright owner are credited and that the original publication in this journal is cited, in accordance with accepted academic practice. No use, distribution or reproduction is permitted which does not comply with these terms. 